Groups Geom. Dyn. 6 (2012), 619-638

DOI $10.4171 / \mathrm{GGD} / 167$
Groups, Geometry, and Dynamics

(C) European Mathematical Society

\title{
On the asymptotics of visible elements and homogeneous equations in surface groups
}

\author{
Yago Antolín, Laura Ciobanu and Noèlia Viles
}

\begin{abstract}
Let $F$ be a group whose abelianization is $\mathbb{Z}^{k}, k \geq 2$. An element of $F$ is called visible if its image in the abelianization is visible, that is, the greatest common divisor of its coordinates is 1 .

In this paper we compute three types of densities, annular, even and odd spherical, of visible elements in surface groups. We then use our results to show that the probability of a homogeneous equation in a surface group to have solutions is neither 0 nor 1 , as the lengths of the right- and left-hand side of the equation go to infinity.
\end{abstract}

Mathematics Subject Classification (2010). 20E05, 68Q25.

Keywords. Free groups, surface groups, equations, visible elements, asymptotic behavior.

\section{Introduction}

Let $F$ be a group whose abelianization is $\mathbb{Z}^{k}$, with $k \geq 2$. An element of $F$ is called visible with respect to a basis of $\mathbb{Z}^{k}$ if its image in the abelianization is visible, that is, the greatest common divisor of its coordinates is 1 . Being visible is, in fact, independent of the basis of $\mathbb{Z}^{k}$ (see Definition 2.3), and we therefore omit the references to the basis henceforth.

Let $\Sigma$ be a compact connected orientable surface of genus $r, r \geq 2$. If $\Sigma$ has no boundary, then a presentation for the fundamental group of $\Sigma$, which we call the surface group of genus $r$, is $\left\langle a_{1}, b_{1}, \ldots, a_{r}, b_{r} \mid\left[a_{1}, b_{1}\right] \ldots\left[a_{r}, b_{r}\right]\right\rangle$. If $\Sigma$ has boundary, then the fundamental group of $\Sigma$ is simply a free group of finite rank. For a group $G$, a positive integer $n$, and a fixed generating set $A$, one defines the sphere of radius $n$ to be the set of elements in $G$ of length $n$, with respect to $A$. Then the spherical density of a set $S$ of elements in $G$ measures the proportion of elements of length $n$ in $S$ in the sphere of radius $n$ as $n$ goes to infinity (see Section 2). The annular density of a set $S$ records the proportions of $S$ in two successive spheres.

While the spherical density of visible elements does not exist for the groups we consider, one can instead look at the 'odd spherical density' and 'even spherical density' of visible elements of odd and even length, respectively. In this paper we 
compute the annular, odd and even spherical densities of visible elements in a class of groups containing the surface groups of compact connected orientable surfaces, with or without boundary. In [9] the annular density of visible elements was computed for all free groups of finite rank ([9], Theorem A), and odd and even spherical density values were also given for the free group of rank two ([9], Theorem 3.7). Since the limits we obtain are different from 0 and from 1 , this shows that visible elements form a set of intermediate density in the groups we study. Intermediate density of sets in groups has been displayed for the first time in [9] for visible and test elements. Then Dunfield and Thurston ([6]) proved that for a random 2-generator one-relator group the property that this group splits as a mapping torus of a free group automorphism is 'intermediate'. However, this tends to be a relatively rare behaviour for many combinatorial and algebraic properties encountered in group theory and most of the properties studied so far in the literature (see [10]) turn out to be negligible or generic, that is, with density equal to 0 or 1 , respectively. For example, in a finitely generated free group the set of all powers is negligible, as is the set of primitive elements, any subgroup of finite index or the union of all proper free factors ([1], [14]). On the other hand, the set of all words whose symmetrizations satisfy the $C^{\prime}\left(\frac{1}{6}\right)$ small cancellation condition, or the set of words with nontrivial images in the abelianization are generic ([1], [14]).

We would also like to mention the results of [11], where densities of sets of conjugacy classes in free and surface groups are investigated. More precisely, the density considered in [11] is the asymptotic density of sets of root-free conjugacy classes of hyperbolic elements in surface groups, and for free groups, the density is similar to the annular density but records the proportion in two successive balls instead of two successive spheres.

A consequence of our results is the fact that the solvability of homogeneous equations in the class of groups we study is a non-negligible and non-generic property. Let $G$ be a finitely generated group, $A$ a fixed generating set, and $X=\left\{X_{1}, \ldots, X_{n}\right\}$, $n \geq 1$, a set of variables. An equation in variables $X_{1}, \ldots, X_{n}$ with coefficients $g_{1}, \ldots, g_{m+1}$ in $G$ is a formal expression given by

$$
g_{1} X_{i_{1}}^{\varepsilon_{1}} g_{2} X_{i_{2}}^{\varepsilon_{2}} \ldots X_{i_{m}}^{\varepsilon_{m}} g_{m+1}=1,
$$

where $m \geq 1, \varepsilon_{j} \in\{1,-1\}$ for all $1 \leq j \leq m$, and $i_{j} \in\{1, \ldots, n\}$. An equation is homogeneous if the variables are on the left-hand side of the equation and the constants are on the right-hand side of the equation,

$$
X_{i_{1}}^{\varepsilon_{1}} X_{i_{2}}^{\varepsilon_{2}} \ldots X_{i_{m}}^{\varepsilon_{m}}=w,
$$

where $w \in G$. We say that the equation (1.1) is a homogeneous equation of type $\left(m,|w|_{A}\right)$ or an $\left(m,|w|_{A}\right)$-homogeneous equation, where $|w|_{A}$ denotes the length of $w$ with respect to $A$.

We will be interested in the asymptotic behavior of $\left(m,|w|_{A}\right)$-homogeneous equations when $G$ is a surface or a free group and $m$ and $|w|_{A}$ go to infinity. Our study of 
the asymptotics of homogeneous equations was motivated by two related questions: firstly, how often does a homogeneous equation in a free or surface group have solutions, and secondly, how likely is it for two random words $u$ and $v$ in the group to have that $v$ is an endomorphic image of $u$ ? The second question asks what the 'generic' answer to the endomorphism problem in free groups is (see [5] for more on the endomorphism problem) and was also inspired by the work of Kapovich, Schupp and Shpilrain ([10]). They show that the probability of two elements $u$ and $v$ in $F_{k}$ to be in the same automorphic orbit is 0 as the lengths of $u$ and $v$ go to infinity. The following paragraph clarifies the relation between the two questions.

Suppose that $z\left(X_{1}, \ldots, X_{n}\right)$ is the word in $X_{1}, \ldots, X_{n}$ representing the left-hand side of (1.1), i.e., $z\left(X_{1}, \ldots, X_{n}\right)=X_{i_{1}}^{\varepsilon_{1}} X_{i_{2}}^{\varepsilon_{2}} \ldots X_{i_{m}}^{\varepsilon_{m}}$. Let $F_{n}$ be the free group of rank $n$ on generators $x_{1}, \ldots, x_{n}$. Notice that the equation (1.1) has solutions if and only if there exists an homomorphism $\phi: F_{n} \rightarrow G$ such that $\phi\left(z\left(x_{1}, \ldots, x_{n}\right)\right)=w$, where $z$ is written in the generators $x_{1}, \ldots, x_{n}$. The following ratios quantify the pairs of elements of the form $(z, w)$.

Definitions 1.1. Let $F, G$ be countable groups and $l_{F}: F \rightarrow \mathbb{N}$ and $l_{G}: G \rightarrow \mathbb{N}$ be length functions as defined in Definition 2.1.

(1) The $(s, t)$-mapping ratio $e_{\rho}(F, G, s, t)$ is the ratio of the pairs of elements $(f, g) \in F \times G$ such that $l_{F}(f) \leq s, l_{G}(g) \leq t$ and with the property that $g$ is a homomorphic image of $f$ among all pairs $(f, g) \in F \times G$ with $l_{F}(f) \leq s, l_{G}(g) \leq t$, that is,

$$
\begin{aligned}
& e_{\rho}(F, G, s, t) \\
& =\frac{\sharp\left\{(f, g) \in F \times G: l_{F}(f) \leq s, l_{G}(g) \leq t, \phi(f)=g \text { for some } \phi \in \operatorname{Hom}(F, G)\right\}}{\sharp\left\{(f, g) \in F \times G: l_{F}(f) \leq s, l_{G}(g) \leq t\right\}} .
\end{aligned}
$$

(2) The spherical $(s, t)$-mapping ratio $e_{\gamma}(F, G, s, t)$ is the ratio of the pairs of elements $(f, g) \in F \times G$ such that $l_{F}(f)=s, l_{G}(g)=t$ and with the property that $g$ is a homomorphic image of $f$ among all pairs $(f, g) \in F \times G$ with $l_{F}(f)=s$, $l_{G}(g)=t$, that is,

$$
\begin{aligned}
& e_{\gamma}(F, G, s, t) \\
& =\frac{\sharp\left\{(f, g) \in F \times G: l_{F}(f)=s, l_{G}(g)=t, \phi(f)=g \text { for some } \phi \in \operatorname{Hom}(F, G)\right\}}{\sharp\left\{(f, g) \in F \times G: l_{F}(f)=s, l_{G}(g)=t\right\}} .
\end{aligned}
$$

In Section 3 we will study the asymptotic behavior of the $(s, t)$-mapping ratio $e_{\rho}(F, G, s, t)$ for $F$ and $G$ free abelian groups with $l_{G}$ and $l_{F}$ being the restriction of the $\|\cdot\|_{p}$ norm, $1 \leq p \leq \infty$. We will show that the limit of $e_{\rho}(F, G, s, t)$, as $s$ and $t$ go to infinity, is neither 0 nor 1 . The computation of the asymptotic behavior of this ratio is based on the densities of visible elements in a free abelian group. In Section 4 we study the annular, even and odd spherical densities of visible elements in free and surface groups: 
Corollary 4.11. Let $k \geq 2$ and let $F$ be a free group of rank $k$ or a surface group of genus $k$. Let $A$ be the symmetric generating set of $F, r$ the rank of the abelianization, and $V_{1}$ the set of visible elements in $F$. Then

(i) $\lim _{m \rightarrow \infty} \frac{\gamma_{A}\left(2 m, V_{1}\right)}{\gamma_{A}(2 m, F)}=\frac{2^{r}-2}{\left(2^{r}-1\right) \zeta(r)}$,

(ii) $\lim _{m \rightarrow \infty} \frac{\gamma_{A}\left(2 m-1, V_{1}\right)}{\gamma_{A}(2 m-1, F)}=\frac{2^{r}}{\left(2^{r}-1\right) \zeta(r)}$,

where $\gamma_{A}(n, S)$ is the number of elements of length $n$ in the set $S \subseteq F$, and $\zeta$ is Euler's $\zeta$ function.

We obtain our main result in Theorem 4.10, which relates the densities of visible points in surface and free groups with the densities in the abelianization. In Section 5 we study the asymptotic behavior of the spherical $(s, t)$-mapping ratio $e_{\gamma}(F, G, s, t)$ when $F$ and $G$ are free or surface groups. We exploit the connection of $e_{\gamma}(F, G, s, t)$ with $e_{\rho}\left(F_{\mathrm{ab}}, G_{\mathrm{ab}}, s, t\right)$ to obtain upper and lower bounds for $e_{\gamma}$ :

Theorem 5.1. Let $G_{k}$ and $G_{n}$ be free or surface groups and let $A, B$ be their respective generating sets. Let $r(k)$ and $r(n)$ denote the ranks of the abelianization of $G_{k}$ and $G_{n}$, respectively. Let $\varepsilon, \delta \in\{0,1\}$ and $\zeta$ be Euler's $\zeta$ function. Then the following inequalities hold:

$$
\begin{aligned}
& \frac{2^{r(n)}-2(1-\varepsilon)}{\left(2^{r(n)}-1\right) \zeta(r(n))} \leq \liminf _{s \rightarrow \infty, t \rightarrow \infty} e_{\gamma}\left(G_{n}, G_{k}, 2 s+\varepsilon, 2 t+\delta\right), \\
& \limsup _{s \rightarrow \infty, t \rightarrow \infty} e_{\gamma}\left(G_{n}, G_{k}, 2 s+\varepsilon, 2 t+\delta\right) \\
& \quad \leq 1-\frac{2^{r(k)}-2(1-\delta)}{\left(2^{r(k)}-1\right) \zeta(r(k))}\left(1-\frac{2^{r(n)}-2(1-\varepsilon)}{\left(2^{r(n)}-1\right) \zeta(r(n))}\right) .
\end{aligned}
$$

As a corollary, we obtain that the probability of an $(s, t)$-homogeneous equation in a surface group to be satisfiable is neither 0 nor 1 as $s, t$ go to infinity (Corollary 5.2).

The results developed in this paper lend themselves to generalizations. For example, one can extend Theorem 4.10 to those strongly Markov groups whose abelianization is free abelian by using Sharp's Corollary 2.1 [13]. One may try to generalize our results to word-hyperbolic groups, which are strongly Markov. However, they could have finite abelianization, and hence no homomorphism onto $\mathbb{Z}$. In [3], Calegari and Fujiwara deal with this situation by considering quasimorphisms to $\mathbb{Z}$ which are, informally, homomorphisms up to bounded error. The central limit theorem of Calegary and Fujiwara may be used to compute densities in hyperbolic groups of sets related to these quasimorphisms.

The asymptotic density of satisfiable homogeneous and one-variable equations in free groups has recently been considered by B. Gilman, A. Miasnikov and V. Roman'kov in [7], who also study the asymptotic behavior of equations in free abelian and free nilpotent groups in [8]. 
On the asymptotics of visible elements and homogeneous equations in surface groups 623

\section{Notation}

Definitions 2.1. Let $F$ be a finitely generated group, and let $A$ be a finite generating set of $F$. If $w \in F$, then $|w|_{A}$ denotes the length of the shortest word in $A^{ \pm 1}$ representing $w$.

For $1 \leq p \leq \infty$, let $l_{p}: \mathbb{Z}^{r} \rightarrow \mathbb{R}$ denote the restriction to $\mathbb{Z}^{r}$ of the $\|\cdot\|_{p}$-norm from $\mathbb{R}^{r}$.

A length function for a set $S$ is a function $l: S \rightarrow \mathbb{N}$ such that, for every $n \in \mathbb{N}$, the set $l^{-1}(\{0,1,2, \ldots, n\})$ is finite. The functions $|\cdot|_{A}$ and $l_{p}$ are examples of length functions in $F$ and $\mathbb{Z}^{r}$.

Definitions 2.2. Let $F$ be a group (or more generally, a set) and $l_{F}: F \rightarrow \mathbb{N}$ a length function.

(1) Let $S \subseteq F$ and $n \geq 0$. Then

$$
\rho_{l_{F}}(n, S)=\sharp\left\{x \in S: l_{F}(x) \leq n\right\}
$$

and

$$
\gamma_{l_{F}}(n, S)=\sharp\left\{x \in S: l_{F}(x)=n\right\}
$$

denote the cardinality of the intersection of $S$ with the ball and sphere of radius $n$ in $F$, respectively.

(2) Let $S \subseteq F$. The asymptotic density of $S$ in $F$ is

$$
\bar{\rho}_{l_{F}}(S)=\limsup _{n \rightarrow \infty} \frac{\rho_{l_{F}}(n, S)}{\rho_{l_{F}}(n, F)} .
$$

If the limit exists, then we denote it by $\rho_{l_{F}}(S)$ and we call it the strict asymptotic density.

(3) Let $S \subseteq F$. The spherical density of $S$ in $F$ is

$$
\bar{\gamma}_{l_{F}}(S)=\limsup _{n \rightarrow \infty} \frac{\gamma_{l_{F}}(n, S)}{\gamma_{l_{F}}(n, F)} .
$$

If the limit exists, then we denote it by $\gamma_{l_{F}}(S)$ and we call it the strict spherical density.

(4) Let $S \subseteq F$. The annular density of $S$ in $F$ is

$$
\bar{\sigma}_{l_{F}}(S)=\limsup _{n \rightarrow \infty} \frac{1}{2}\left(\frac{\sharp\left\{x \in S: l_{F}(x)=n-1\right\}}{\sharp\left\{x \in F: l_{F}(x)=n-1\right\}}+\frac{\sharp\left\{x \in S: l_{F}(x)=n\right\}}{\sharp\left\{x \in F: l_{F}(x)=n\right\}}\right)
$$

If the limit exists, then we denote it by $\sigma_{l_{F}}(S)$ and we call it the strict annular density.

When $F$ is a group, finitely generated by $A$, and $l_{F}=|\cdot|_{A}$, the word length, we will just write $\rho_{A}, \gamma_{A}$ and $\sigma_{A}$. Similarly if $F=\mathbb{Z}^{r}$ and $l_{F}=l_{p}$, the restriction of the $p$-norm, we will just write $\rho_{p}, \gamma_{p}$ and $\sigma_{p}$. 
Definitions 2.3. For a nonzero element $z \in \mathbb{Z}^{r}$ we denote by $\operatorname{gcd}(z)$ the greatest common divisor of its coordinates. If $z=(0, \ldots, 0) \in \mathbb{Z}^{r}$ we set $\operatorname{gcd}(z)=\infty$. Note that gcd is invariant under the action of $\operatorname{Aut}\left(\mathbb{Z}^{r}\right)=\operatorname{SL}(r, \mathbb{Z})$. Hence, for all $z \in \mathbb{Z}^{r}, \operatorname{gcd}(z)$ does not depend on the basis of $\mathbb{Z}^{r}$.

An element of $z \in \mathbb{Z}^{r}$ is called visible if $\operatorname{gcd}(z)=1$. If $\operatorname{gcd}(z)=t$, then we call the element $t$-visible.

We denote by $F_{\text {ab }}$ the abelianization of the group $F$, that is, $F_{\mathrm{ab}}=F /[F, F]$. Suppose that $F_{\mathrm{ab}}$ is a free abelian group of finite rank and let ab: $F \rightarrow F_{\mathrm{ab}}$ be the abelianization map. We say that an element $f \in F$ is visible (resp. $t$-visible) if $\mathrm{ab}(f)$ is visible (resp. $t$-visible) in $F_{\mathrm{ab}}$.

\section{Densities of visible elements in $\mathbb{Z}^{r}$}

Let $r \geq 2$ be an integer and let $U_{t}$ denote the set of all $t$-visible elements in $\mathbb{Z}^{r}$. For a complex number $k$, recall that the Riemann zeta function is given by

$$
\zeta(k)=\sum_{n=1}^{\infty} \frac{1}{n^{k}}, \quad \operatorname{Re}(k)>1 .
$$

A classical result in number theory provides the value for the strict asymptotic density of $t$-visible elements in $\mathbb{Z}^{r}$.

Proposition 3.1 ([4]). For any integer $t \geq 1$,

$$
\rho_{\infty}\left(U_{t}\right)=\frac{1}{t^{r} \zeta(r)}
$$

By [9], Theorem A (1), one can substitute $\rho_{\infty}$ by $\rho_{p}$ for the sets $U_{t}$ :

Proposition 3.2 ([9], Theorem A (1)). For any integer $t \geq 1$ and any $p, 1 \leq p \leq \infty$,

$$
\rho_{p}\left(U_{t}\right)=\rho_{\infty}\left(U_{t}\right) .
$$

The following lemma shows that homomorphisms between groups with free abelian abelianization (of finite rank) send $t$-visible elements to $(\mathrm{tm}$ )-visible elements, where $t, m$ are positive integers. The second part of the lemma shows that a visible element in a group can be mapped to any element via a homomorphism.

Lemma 3.3. Let $F, G$ be groups whose abelianization is free abelian of finite rank. Let $f \in F$.

(i) Let $\phi: F \rightarrow G$ be a group homomorphism. Then $\operatorname{gcd}(\operatorname{ab}(\phi(f)))$ is a multiple of $\operatorname{gcd}(\mathrm{ab}(f))$. In particular, if $\operatorname{gcd}(\mathrm{ab}(f))=\infty$, then $\operatorname{gcd}(\mathrm{ab}(\phi(f)))=\infty$. 
On the asymptotics of visible elements and homogeneous equations in surface groups 625

(ii) If, moreover $\operatorname{gcd}(\mathrm{ab}(f))=1$, then for any element $g$ in $G$ there exists an homomorphism $\phi: F \rightarrow G$ such that $\phi(f)=g$.

Proof. Let $n$ be the rank of $F_{\mathrm{ab}}$ and let $\left\{e_{1}, \ldots, e_{n}\right\}$ be a basis of $F_{\mathrm{ab}}$. For $f \in F_{\mathrm{ab}}$, we denote by $(f)_{i}$ the $i$ th coordinate of $f$ with respect to the basis. That is, $f=$ $(f)_{1} e_{1}+\cdots+(f)_{n} e_{n}$.

(i) Let $g=\phi(f)$. Then $(\mathrm{ab}(g))_{j}=\sum_{i=1}^{n}(\mathrm{ab}(f))_{i}\left(\phi\left(e_{i}\right)\right)_{j}$.

Thus each $(\mathrm{ab}(g))_{j}$ is a multiple of $\operatorname{gcd}(\mathrm{ab}(f))$ since each $(\mathrm{ab}(f))_{i}$ is a multiple of $\operatorname{gcd}(\mathrm{ab}(f))$.

(ii) Since $\operatorname{gcd}(\mathrm{ab}(f))=1, \operatorname{gcd}\left((\operatorname{ab}(f))_{1}, \ldots,(\mathrm{ab}(f))_{n}\right)=1$ and therefore there exist integers $p_{1}, \ldots, p_{n}$ such that $\sum_{i=1}^{n}(\mathrm{ab}(f))_{i} p_{i}=1$. Consider the homomorphism $\psi_{1}: F_{\mathrm{ab}} \rightarrow\langle x \mid\rangle$ which sends $e_{i}$ to $x^{p_{i}}$ for all $1 \leq i \leq n$. It follows that $\psi_{1}(\mathrm{ab}(f))=x$. Let $\psi_{2}:\langle x \mid\rangle \rightarrow G$ be any homomorphism sending $x$ to $g$. This shows that the composition of ab, $\psi_{1}$ and $\psi_{2}$ produces a homomorphism $\phi: F \rightarrow G$ such that $\phi(f)=g$.

Corollary 3.4. Let $\mathbb{Z}^{n}$ and $\mathbb{Z}^{k}$ be the free abelian groups of ranks $n$ and $k$, respectively. Then the following inequalities hold with respect to $l_{p}$ for $1 \leq p \leq \infty$ :

$$
\begin{gathered}
\frac{1}{\zeta(n)} \leq \liminf _{s \rightarrow \infty, t \rightarrow \infty} e_{\rho}\left(\mathbb{Z}^{n}, \mathbb{Z}^{k}, s, t\right), \\
\limsup _{s \rightarrow \infty, t \rightarrow \infty} e_{\rho}\left(\mathbb{Z}^{n}, \mathbb{Z}^{k}, s, t\right) \leq 1-\frac{1}{\zeta(k)}\left(1-\frac{1}{\zeta(n)}\right) .
\end{gathered}
$$

Proof. We fix some $p, 1 \leq p \leq \infty$. Let $e_{\mathrm{ab}}(s, t):=e_{\rho}\left(\mathbb{Z}^{n}, \mathbb{Z}^{k}, s, t\right)$ with respect the length $l_{p}$ and let $|u|=l_{p}(u)$.

By Lemma 3.3 (ii)

$$
\begin{aligned}
e_{\mathrm{ab}}(s, t) & \geq \frac{\sharp\left\{(u, v) \in \mathbb{Z}^{n} \times \mathbb{Z}^{k}:|u| \leq s,|v| \leq t, \operatorname{gcd}(u)=1\right\}}{\rho_{p}\left(s, \mathbb{Z}^{n}\right) \rho_{p}\left(t, \mathbb{Z}^{k}\right)} \\
& =\frac{\sharp\left\{u \in \mathbb{Z}^{n}:|u| \leq s, \operatorname{gcd}(u)=1\right\}}{\rho_{p}\left(s, \mathbb{Z}^{n}\right)} .
\end{aligned}
$$

Taking limits, we obtain (3.1) by Propositions 3.1 and 3.2.

By Lemma 3.3 (i),

$$
\begin{gathered}
e_{\mathrm{ab}}(s, t) \leq 1-\frac{\sharp\left\{(u, v) \in \mathbb{Z}^{n} \times \mathbb{Z}^{k}:|u| \leq s,|v| \leq t, \operatorname{gcd}(u) \neq 1, \operatorname{gcd}(v)=1\right\}}{\rho_{p}\left(s, \mathbb{Z}^{n}\right) \rho_{p}\left(t, \mathbb{Z}^{k}\right)} \\
=1-\left(1-\frac{\sharp\left\{u \in \mathbb{Z}^{n}:|u| \leq s, \operatorname{gcd}(u)=1\right\}}{\rho_{p}\left(s, \mathbb{Z}^{n}\right)}\right) \\
\cdot \frac{\sharp\left\{v \in \mathbb{Z}^{k}:|v| \leq t, \operatorname{gcd}(v)=1\right\}}{\rho_{p}\left(t, \mathbb{Z}^{k}\right)} .
\end{gathered}
$$

Taking limits, we obtain (3.2) by Propositions 3.1 and 3.2. 
One of the key ingredients needed to extend the previous result to the analogue for surface groups is determining the asymptotic density of elements of even length in $\mathbb{Z}^{k}$. This was done in [9], Proposition 3.6, for $k=2$, and we now compute the value for a general $k$.

Proposition 3.5. Let $k \geq 2$, and let $U_{1}^{\mathrm{ev}}=\left\{z \in U_{1}: l_{1}(z)\right.$ is even $\}$ denote the set of visible elements of even length in $\mathbb{Z}^{k}$. Then

$$
\rho_{\infty}\left(U_{1}^{\mathrm{ev}}\right)=\frac{2^{k-1}-1}{2^{k}-1} \rho_{\infty}\left(U_{1}\right)=\frac{2^{k-1}-1}{\left(2^{k}-1\right) \zeta(k)} .
$$

Proof. Let $n$ be a positive integer and let $[0, n]=\{0,1, \ldots, n\}$. For $X_{1}, \ldots, X_{k} \in$ $\{\mathscr{A}, \mathcal{O}, \mathscr{E}\}$ we denote by $X_{1} X_{2} \ldots X_{k}(n)$ the number of all $z=\left(z_{1}, \ldots, z_{k}\right) \in U_{1}$ such that $z_{i} \in[0, n]$ and the parity of $z_{i}$ is $X_{i}$. Here $\mathcal{A}$ stands for "any", $\mathcal{E}$ stands for "even" and $\mathcal{O}$ stands for "odd".

We will use the convention $\underbrace{X \ldots X}_{\mathrm{k} \text { times }}=X^{k}$ for any $X \in\{\mathcal{A}, \mathcal{O}, \mathcal{E}\}$ and $k \geq 1$. Note that $X_{1} X_{2} \ldots X_{k}(n)=X_{s(1)} X_{s(2)} \ldots X_{s(k)}(n)$, for any permutation $s$ of $\{1, \ldots, k\}$, and that $\mathcal{E}^{k}(n)=0$ for any $k, n \geq 1$.

The total number of elements in $U_{1}$ in $[0, n]^{k}$ is

$$
\mathcal{A}^{k}(n)=\sum_{i=1}^{k}\left(\begin{array}{l}
k \\
i
\end{array}\right) \mathcal{E}^{k-i} \mathcal{O}^{i}(n) .
$$

Let $U_{1}^{\mathrm{ev}}(n)$ be the set $U_{1}^{\mathrm{ev}} \cap[0, n]^{k}$. Then

$$
\left|U_{1}^{\mathrm{ev}}(n)\right|=\sum_{i=1}^{\left[\frac{k}{2}\right]}\left(\begin{array}{c}
k \\
2 i
\end{array}\right) \mathcal{E}^{k-2 i} \mathcal{O}^{2 i}(n) .
$$

We claim that

$$
\mathcal{E}^{k-i} \mathcal{O}^{i}(n)=\mathcal{O}^{k}(n)+o\left(n^{k}\right) \text { for all } 1 \leq i \leq k .
$$

Assume first that (3.5) holds. From (3.4) and (3.5) we get

$$
\left|U_{1}^{\mathrm{ev}}(n)\right|=\sum_{i=1}^{\left[\frac{k}{2}\right]}\left(\begin{array}{c}
k \\
2 i
\end{array}\right) \mathcal{O}^{k}(n)+o\left(n^{k}\right),
$$

and since $\sum_{i=1}^{\left[\frac{k}{2}\right]}\left(\begin{array}{c}k \\ 2 i\end{array}\right)=2^{k-1}-1$, we get

$$
\left|U_{1}^{\mathrm{ev}}(n)\right|=\left(2^{k-1}-1\right) \mathcal{O}^{k}(n)+o\left(n^{k}\right) .
$$


On the asymptotics of visible elements and homogeneous equations in surface groups 627

Since $\sum_{i=1}^{k}\left(\begin{array}{l}k \\ i\end{array}\right)=2^{k}-1$, from (3.3) and (3.5) we obtain

$$
\mathcal{O}^{k}(n)\left(2^{k}-1\right)=\mathcal{A}^{k}(n)+o\left(n^{k}\right),
$$

and hence

$$
\left|U_{1}^{\mathrm{ev}}(n)\right|=\frac{2^{k-1}-1}{2^{k}-1} \mathcal{A}^{k}(n)+o\left(n^{k}\right) .
$$

Since $\rho_{\infty}\left(U_{1}\right)=\lim _{n \rightarrow \infty} \frac{\mathcal{A}^{k}(n)}{n^{k}}=\frac{1}{\zeta(k)}$, we get

$$
\begin{aligned}
\rho_{\infty}\left(U_{1}^{\mathrm{ev}}\right) & =\limsup _{n \rightarrow \infty} \frac{\left|U_{1}^{\mathrm{ev}}(n)\right|}{n^{k}} \\
& =\lim _{n \rightarrow \infty} \frac{\frac{2^{k-1}-1}{2^{k}-1} \mathcal{A}^{k}(n)+o\left(n^{k}\right)}{n^{k}} \\
& =\frac{2^{k-1}-1}{2^{k}-1} \rho_{\infty}\left(U_{1}\right)=\frac{2^{k-1}-1}{\left(2^{k}-1\right) \zeta(k)} .
\end{aligned}
$$

This completes the proof of the proposition. We now show (3.5). Notice first that

$$
\mathcal{O}^{i} \mathcal{E}^{k-i-1} \mathcal{A}(n)=\mathcal{O}^{i} \mathcal{E}^{k-i}(n)+\mathcal{O}^{i+1} \mathcal{E}^{k-i-1}(n) .
$$

Hence it is enough to show

$$
\mathcal{O}^{i} \mathcal{E}^{k-i-1} \mathcal{A}(n)=2 \mathcal{O}^{i} \mathcal{E}^{k-i}(n)+o\left(n^{k}\right) \text { for all } 1 \leq i \leq k .
$$

Let $\mu: \mathbb{N} \rightarrow\{-1,0,1\}$ denote the Möbius function and recall that $\sum_{d \mid n} \mu(d)$ is equal to 1 , if $n=1$ and 0 otherwise. Hence

$$
\mathcal{O}^{i} \mathcal{E}^{k-i-1} \mathcal{A}(n)=\sum_{\substack{0 \leq x_{j} \leq n, 2 \nmid x_{j} \\ j=1, \ldots, i}} \sum_{\substack{0 \leq x_{j} \leq n, 2 \mid x_{j} \\ j=i+1, \ldots, k-1}} \sum_{0 \leq x_{k} \leq n} \sum_{d \mid \operatorname{gcd}\left(x_{1}, \ldots, x_{k}\right)} \mu(d)
$$

and

$$
\mathcal{O}^{i} \mathcal{E}^{k-i}(n)=\sum_{\substack{0 \leq x_{j} \leq n, 2 \nmid x_{j} \\ j=1, \ldots, i}} \sum_{\substack{0 \leq x_{j} \leq n, 2 \mid x_{j} \\ j=i+1, \ldots, k}} \sum_{d \mid \operatorname{gcd}\left(x_{1}, \ldots, x_{k}\right)} \mu(d) .
$$

Now we switch the order in the summation. We rearrange the terms depending on $d \mid \operatorname{gcd}\left(x_{1}, \ldots, x_{k}\right)$, writing $x_{i}=y_{i} d$. Since there is an odd coordinate, $2 \nmid d$. We obtain

$$
\mathcal{O}^{i} \mathcal{E}^{k-i-1} \mathcal{A}(n)=\sum_{2 \nmid d} \mu(d) \sum_{\substack{0 \leq y_{j} \leq n / d, 2 \nmid y_{j} \\ j=1, \ldots, i}} \sum_{\substack{0 \leq y_{j} \leq n / d, 2 \mid y_{j} \\ j=i+1, \ldots, k-1}} \sum_{y_{k} \leq n / d} 1
$$

and

$$
\mathcal{O}^{i} \mathcal{E}^{k-i}(n)=\sum_{2 \nmid d} \mu(d) \sum_{\substack{0 \leq y_{j} \leq n / d, 2 \nmid y_{j} \\ j=1, \ldots, i}} \sum_{\substack{0 \leq y_{j} \leq n / d, 2 \mid y_{j} \\ j=i+1, \ldots, k}} 1
$$


Hence $\mathcal{O}^{i} \mathcal{E}^{k-i-1} \mathcal{A}(n)-2 \mathcal{O}^{i} \mathcal{E}^{k-i}(n)$ is equal to

$$
\sum_{2 \nmid d} \mu(d) \sum_{\substack{0 \leq y_{j} \leq n / d, 2 \nmid y_{j} \\ j=1, \ldots, i}} \sum_{\substack{0 \leq y_{j} \leq n / d, 2 \mid y_{j} \\ j=i+1, \ldots, k-1}}\left(\left[\frac{n}{d}\right]-2\left[\frac{n}{2 d}\right]\right) .
$$

The term in parenthesis is either 0 or 1 , and it is always 0 for $d>n$. Thus the asymptotic behavior of (3.6) is of type

$$
\begin{aligned}
O\left(\sum_{d \leq n} \sum_{\substack{0 \leq y_{j} \leq n / d \\
j=1, \ldots, k-1}} 1\right) & \subseteq O\left(\sum_{d=1}^{n}(n / d)^{k-1}\right) \\
& =O\left(n^{k-1}\left(\frac{1}{k-2}-\frac{1}{(k-2) n^{k-2}}\right)\right) \\
& =O\left(n^{k-1}\right) \subset o\left(n^{k}\right) .
\end{aligned}
$$

\section{Densities of visible elements in surface groups}

The main result of this section is an extension of [9], Theorem A, that allows us to compute densities of visible elements in free and surface groups. We need to fix some notation.

Notation 4.1. For $k \geqslant 2$, we denote by $F_{k}$ the free group of rank $k$ and by $S_{k}$ the surface group of genus $k$.

We will work with the standard presentation for $F_{k}$,

$$
\left\langle a_{1}, \ldots, a_{k} \mid\right\rangle
$$

and let $A=\left\{a_{1}, \ldots, a_{k}\right\}^{ \pm 1}$.

A presentation for $S_{k}$ has the form

$$
\left\langle a_{1}, b_{1}, \ldots, a_{k}, b_{k} \mid\left[a_{1}, b_{1}\right] \ldots\left[a_{k}, b_{k}\right]\right\rangle .
$$

In this case we let $A=\left\{a_{1}, b_{1}, \ldots, a_{k}, b_{k}\right\}^{ \pm 1}$.

Let $r$ denote the rank of the abelianization, that is, $r=k$ for $F_{k}$, and $r=2 k$ for $S_{k}$.

Our main result is based on the following local limit theorem of Sharp in [13].

Theorem 4.2 (see Theorems 1, 3, 4 in [13]). Let $F$ be $F_{k}$ or $S_{k}$, and $A$ and $r$ be the corresponding generating set and rank of the abelianization of $F$, as in Notation 4.1.

Let $\mathrm{ab}: F \rightarrow \mathbb{Z}^{r}$ be the abelianization map. Then there exists a symmetric positive definite real matrix $D$ such that, uniformly in $\alpha \in \mathbb{Z}^{r}$,

$$
\begin{gathered}
\lim _{n \rightarrow \infty} \mid(\operatorname{det} D)^{1 / 2} n^{r / 2}\left(\frac{\gamma_{A}\left(n, \mathrm{ab}^{-1}(\alpha)\right)}{\gamma_{A}(n, F)}+\frac{\gamma_{A}\left(n+1, \mathrm{ab}^{-1}(\alpha)\right)}{\gamma_{A}(n+1, F)}\right) \\
-\frac{2}{(2 \pi)^{r / 2}} e^{-\left\langle\alpha, D^{-1} \alpha\right\rangle / 2 n} \mid=0 .
\end{gathered}
$$


On the asymptotics of visible elements and homogeneous equations in surface groups 629

Proof. For $F=S_{k}$ this is exactly [13], Theorem 4, with $g=r / 2$. For $F=F_{k}$ and $D$ the diagonal matrix with all entries equal to $\sigma^{2}$, one obtains exactly [13], Theorem 1.

Since the proof of the main theorem of this section does not use the fact that $F$ is a free or surface group, but only the conclusions of Theorem 4.2, we will fix the following hypothesis.

Hypothesis 4.3. Let $F$ be a group generated by a finite set $A$ such that $F_{\mathrm{ab}} \cong \mathbb{Z}^{r}$ and $D$ be a symmetric positive definite real matrix such that the limit (4.1) goes to zero uniformly in $\alpha \in \mathbb{Z}^{r}$.

By Theorem 4.2, the free group $F_{k}$ and the surface group $S_{k}$ of Notation 4.1 satisfy Hypothesis 4.3.

Definition 4.4. Let $G_{r}$ be the set of all $M \in \operatorname{SL}(r, \mathbb{Z})$ such that $M=I_{r}$ in $\operatorname{SL}(r, \mathbb{Z} / 2 \mathbb{Z})$. Then $G_{r}$ is a finite-index subgroup of $\operatorname{SL}(r, \mathbb{Z})$.

Definition 4.5. We say that a bounded open subset of $\mathbb{R}^{r}$ is nice if its boundary is piecewise smooth.

Proposition 4.6 ([9], Proposition 3.3). Let $S \subseteq \mathbb{Z}^{r}$ be a $G_{r}$-invariant subset such that $\delta=\rho_{\infty}(S)$ exists. Let $\Omega \subseteq \mathbb{R}^{r}$ be a nice bounded open set and for $t \in \mathbb{R}, t>0$, let

$$
\mu_{t, S}(\Omega):=\frac{\sharp(S \cap t \Omega)}{t^{r}} .
$$

Then we have

$$
\lim _{t \rightarrow \infty} \mu_{t, S}(\Omega)=\delta \lambda(\Omega),
$$

where $\lambda$ is the Lebesgue measure.

Although [9] indicates that the proof is similar to that of [9], Proposition 2.3, we include here a proof for Proposition 4.6 for the sake of completeness.

Proof. Each $\mu_{t, S}$ can be regarded as a measure on $\mathbb{R}^{r}$. We prove the result by showing that $\mu_{t, S}$ weakly converges to $\delta \lambda$ as $t \rightarrow \infty$.

By Helly's theorem (see, for instance, [2], Theorem 25.9), there exists a sequence $\left\{t_{i}\right\}$ with $\lim _{i \rightarrow \infty} t_{i}=\infty$ such that the sequence $\mu_{t_{1}, S}, \mu_{t_{2}, S}, \ldots$ is weakly convergent to some limiting measure. We now identify this measure by showing that for every convergent subsequence of $\mu_{t_{i}, S}$ the limiting measure is equal to $\delta \lambda$.

Indeed, we assume that $\eta=\left\{t_{i}\right\}$ is a sequence with $\lim _{i \rightarrow \infty} t_{i}=\infty$ such that the sequence $\mu_{t_{i}, S}$ converges to the limiting measure $\mu_{\eta}=\lim _{i \rightarrow \infty} \mu_{t_{i}, S}$. Every $\mu_{t_{i}, S}$ is invariant with respect to the $G_{r}$-action on $\mathbb{R}^{r}$. Therefore the limiting measure $\mu_{\eta}$ is also $G_{r}$-invariant. Moreover, the measures $\mu_{t, S}$ are dominated by the measures $\lambda_{t}$ defined as $\lambda_{t}(\Omega)=\frac{\sharp\left(\mathbb{Z}^{r} \cap t \Omega\right)}{t^{r}}$. 
It is well known that if $\Omega \subseteq \mathbb{R}^{r}$ is a nice bounded open set, then the measures $\lambda_{t}$ converge to the Lebesgue measure $\lambda$. It follows that $\mu_{\eta}$ is absolutely continuous with respect to $\lambda$. It is also known that the natural action of $G_{r}$ on $\mathbb{R}^{r}$ is ergodic with respect to $\lambda$ (see [15] for the proof of ergodicity). Therefore $\mu_{\eta}$ is a constant multiple $c \lambda$ of $\lambda$. The constant $c$ can be computed for a set such as the open unit ball $B$ in the $\|\cdot\|_{\infty}$ norm on $\mathbb{R}^{r}$ defining the length function $l_{\infty}$ on $\mathbb{Z}^{r}$. By assumption we know that

$$
\rho_{\infty}(S)=\lim _{t \rightarrow \infty} \frac{\sharp\left\{z \in \mathbb{Z}^{r}: z \in S \cap t B\right\}}{\sharp\left\{z \in \mathbb{Z}^{r}: z \in t B\right\}}=\delta .
$$

We also have

$$
\lim _{t \rightarrow \infty} \frac{\sharp\left\{z \in \mathbb{Z}^{r}: z \in t B\right\}}{t^{r}}=\lambda(B)
$$

and hence

$$
\begin{aligned}
\lim _{t \rightarrow \infty} \frac{\sharp\left\{z \in \mathbb{Z}^{r}: z \in t B\right\}}{t^{r}} \frac{\sharp\left\{z \in \mathbb{Z}^{r}: z \in S \cap t B\right\}}{\sharp\left\{z \in \mathbb{Z}^{r}: z \in t B\right\}} & =\lim _{t \rightarrow \infty} \frac{\sharp\left\{z \in \mathbb{Z}^{r}: z \in S \cap t B\right\}}{t^{r}} \\
& =\delta \lambda(B) .
\end{aligned}
$$

Therefore $c=\delta$ and $\mu_{\eta}=\delta \lambda$. The above argument shows in fact that every convergent subsequence of $\mu_{t, S}$ converges to $\delta \lambda$ and $\lim _{t \rightarrow \infty} \mu_{t, S}=\delta \lambda$.

Definition 4.7. Let $F$ be a group generated by the finite set $A$ such that $F_{\mathrm{ab}} \cong \mathbb{Z}^{r}$.

For an integer $n \geqslant 1$ and a point $x \in \mathbb{R}^{r}$, let $p_{n}$ be given by

$$
\begin{aligned}
& p_{n}(x) \\
& =\frac{1}{2}\left(\frac{\gamma_{A}(n-1,\{g \in F: \mathrm{ab}(g)=x \sqrt{n}\})}{\gamma_{A}(n-1, F)}+\frac{\gamma_{A}(n,\{g \in F: \mathrm{ab}(g)=x \sqrt{n}\})}{\gamma_{A}(n, F)}\right) .
\end{aligned}
$$

This is a distribution supported on finitely many points of $\frac{1}{\sqrt{n}} \mathbb{Z}^{r}$.

We need the following results from [12], [13] about the sequence of distributions $p_{n}$.

In our context, we need to restate our Hypothesis 4.3

Proposition 4.8 ([12], [13], [9]). Let F, A, $r$ satisfy Hypothesis 4.3. Then there exists a normal distribution $\mathfrak{\Re}$ with density $\mathfrak{n}$ such that:

(a) The sequence of distributions $p_{n}$ converges weakly to $\mathfrak{n}$ and we have

$$
\sup _{x \in \mathbb{Z}^{r / \sqrt{n}}}\left|n^{r / 2} p_{n}(x)-\mathfrak{n}(x)\right| \longrightarrow 0 \quad \text { as } n \rightarrow \infty .
$$

(b) For $c>0$, let $\overline{\Omega_{c}}:=\left\{x \in \mathbb{R}^{r}:\|x\| \geqslant c\right\}$. Then

$$
\lim _{c \rightarrow \infty}\left(\lim _{n \rightarrow \infty} \sum_{x \in \overline{\Omega_{c}}} p_{n}(x)\right)=0 .
$$


On the asymptotics of visible elements and homogeneous equations in surface groups 631

Proof. Let $D$ be the matrix of Hypothesis 4.3, and let $\mathfrak{n}(x)=\frac{e^{-\left\langle x, D^{-1} x\right\rangle / 2}}{(2 \pi)^{r}(\operatorname{det} D)^{1 / 2}}$, the density of a normal distribution $\mathfrak{N}$. Firstly, we prove the limit in (4.3).

After performing some easy computations,

$$
\begin{aligned}
\left|n^{r / 2} p_{n}(x)-\mathfrak{n}(x)\right| & \frac{1}{2(\operatorname{det} D)^{1 / 2}} \mid(\operatorname{det} D)^{1 / 2} n^{r / 2} \\
& \quad \cdot\left(\frac{\gamma_{A}\left(n-1, \mathrm{ab}^{-1}(x \sqrt{n})\right)}{\gamma_{A}(n-1, F)}+\frac{\gamma_{A}\left(n, \mathrm{ab}^{-1}(x \sqrt{n})\right)}{\gamma_{A}(n, F)}\right)-\frac{2}{(2 \pi)^{r}} e^{\left\langle x, D^{-1} x\right\rangle / 2} \mid \\
= & \frac{1}{2(\operatorname{det} D)^{1 / 2}} \mid(\operatorname{det} D)^{1 / 2} n^{r / 2} \\
& \cdot\left(\frac{\gamma_{A}\left(n-1, \mathrm{ab}^{-1}(\alpha)\right)}{\gamma_{A}(n-1, F)}+\frac{\gamma_{A}\left(n, \mathrm{ab}^{-1}(\alpha)\right)}{\gamma_{A}(n, F)}\right)-\frac{2}{(2 \pi)^{r}} e^{-\left\langle\frac{\alpha}{\sqrt{n}}, \frac{D^{-1} \alpha}{\sqrt{n}}\right\rangle / 2} \mid \\
= & \frac{1}{2(\operatorname{det} D)^{1 / 2}} \mid(\operatorname{det} D)^{1 / 2} n^{r / 2} \\
& \cdot\left(\frac{\gamma_{A}\left(n-1, \mathrm{ab}^{-1}(\alpha)\right)}{\gamma_{A}(n-1, F)}+\frac{\gamma_{A}\left(n, \mathrm{ab}^{-1}(\alpha)\right)}{\gamma_{A}(n, F)}\right)-\frac{2}{(2 \pi)^{r}} e^{-\left\langle\alpha, D^{-1} \alpha\right\rangle / 2 n} \mid,
\end{aligned}
$$

using the limit (4.1) of the Hypothesis 4.3 and the fact that this limit is uniform in $\alpha=x \sqrt{n}$, we obtain the desired result.

In order to show that the sequence of probability distributions $\left\{p_{n}\right\}$ converges weakly to $\mathfrak{n}$, we use [2], Theorem 25.8 , that is, it is necessary and sufficient that for every bounded continuous function $f(x)$ on $\mathbb{R}^{r}$ the following holds:

$$
\lim _{n \rightarrow \infty} \int_{\mathbb{R}^{r}} f(x) p_{n}(x) d \lambda(x)=\int_{\mathbb{R}^{r}} f(x) \mathfrak{n}(x) d \lambda(x) .
$$

We can write

$$
\begin{aligned}
& \left|\int_{\mathbb{R}^{r}} f(x) p_{n}(x) d \lambda(x)-\int_{\mathbb{R}^{r}} f(x) \mathfrak{n}(x) d \lambda(x)\right| \\
& \quad=\left|\int_{\mathbb{R}^{r}} f(x)\left(p_{n}(x)-\mathfrak{n}(x)\right) d \lambda(x)\right| \leqslant \int_{\mathbb{R}^{r}}|f(x)|\left|n^{r / 2} p_{n}(x)-\mathfrak{n}(x)\right| d \lambda(x) .
\end{aligned}
$$

Given that $f$ is a bounded continuous function and by the limit (4.3) proved above, the hypothesis of the Dominated Convergence Theorem is satisfied. Applying this last result, we obtain

$$
\left|\int_{\mathbb{R}^{r}} f(x) p_{n}(x) d \lambda(x)-\int_{\mathbb{R}^{r}} f(x) \mathfrak{n}(x) d \lambda(x)\right| \stackrel{n \rightarrow \infty}{\longrightarrow} 0,
$$

and the weak convergence of the sequence $\left\{p_{n}\right\}$ is proved. 
We now prove $(b)$. For $c>0$, let $\Omega_{c}=\left\{x \in \mathbb{R}^{r}:\|x\|<c\right\}$, and denote by $\overline{\Omega_{c}}$ the complement of $\Omega_{c}$. Then, by the weak convergence of the $p_{n}$ to $\mathfrak{n}$, we have that

$$
\begin{aligned}
\lim _{c \rightarrow \infty}\left(\lim _{n \rightarrow \infty} \sum_{x \in \overline{\Omega_{c}}} p_{n}(x)\right) & =\lim _{c \rightarrow \infty}\left(1-\lim _{n \rightarrow \infty} \sum_{x \in \Omega_{c}} p_{n}(x)\right) \\
& =1-\lim _{c \rightarrow \infty} \int_{x \in \Omega_{c}} \mathfrak{n}(x) d \lambda(x)=0 .
\end{aligned}
$$

Theorem 4.9. Let $\Omega \subseteq \mathbb{R}^{r}$ be a nice bounded open set. Let $S \subseteq \mathbb{Z}^{r}$ be a $G_{r}$ invariant subset such that $\delta=\rho_{\infty}(S)$ exists. Then there exists a normal distribution $\mathfrak{N}$ such that

$$
\lim _{n \rightarrow \infty} \sum_{x \in S \cap \sqrt{n} \Omega} p_{n}(x / \sqrt{n})=\delta \Re(\Omega) .
$$

Proof. Note that the proof is the same as that of Theorem 3.4 in [9]. The only difference lies in the use of Proposition 4.6.

There exists a normal $\mathfrak{N}$ distribution with density $\mathfrak{n}$ satisfying the conclusions of Proposition 4.8.

We have

$$
\begin{aligned}
\sum_{x \in S \cap \sqrt{n} \Omega} p_{n}(x / \sqrt{n}) & =\sum_{y \in \frac{1}{\sqrt{n}} S \cap \Omega} p_{n}(y) \\
& =n^{-r / 2} \sum_{y \in \frac{1}{\sqrt{n}} S \cap \Omega} \mathfrak{n}(y)+n^{-r / 2} \sum_{y \in \frac{1}{\sqrt{n}} S \cap \Omega}\left(n^{r / 2} p_{n}(y)-\mathfrak{n}(y)\right) .
\end{aligned}
$$

The local limit theorem of Proposition 4.8 (a) tells us that, as $n \rightarrow \infty$, each summand $n^{-r / 2} p_{n}(y)-\mathfrak{n}(y)$ of the sum in the last line above converges to zero, and hence so does their Cesaro mean.

Using the following convergence of the measures defined in Proposition 4.6,

$$
\lim _{n \rightarrow \infty} \mu_{\sqrt{n}, S}(\Omega)=\delta \lambda(\Omega)
$$

(recall that $\left.\mu_{\sqrt{n}, S}(\Omega):=\frac{\sharp\left(S \cap \sqrt{n}^{n} \Omega\right)}{\sqrt{n}^{r}}\right)$, we have

$$
\lim _{n \rightarrow \infty} \sum_{x \in S \cap \sqrt{n} \Omega} \frac{1}{(\sqrt{n})^{r}} \mathfrak{n}(x / \sqrt{n})=\int_{\Omega} \mathfrak{n}(y) \delta d \lambda(y)=\delta \Re(\Omega) .
$$

We obtain the main result of this section by basically following [9], Theorem A. Our theorem provides the formula for the 'spherical densities' of visible elements in groups that satisfy Hypothesis 4.3, which include free groups of all finite ranks and surface groups. 
On the asymptotics of visible elements and homogeneous equations in surface groups 633

Theorem 4.10 (see also [9], Theorem A). Let F, A, r satisfy Hypothesis 4.3, let $S \subseteq \mathbb{Z}^{r}$ be a $G_{r}$-invariant subset and $\widetilde{S}=\mathrm{ab}^{-1}(S)$.

(i) The strict annular density $\sigma_{A}(\widetilde{S})$ exists and, moreover, $\sigma_{A}(\tilde{S})=\rho_{\infty}(S)$.

(ii) Let $U_{1}$ denote the set of visible elements in $\mathbb{Z}^{r}$ and $V_{1}=\mathrm{ab}^{-1}\left(U_{1}\right)$ denote the visible elements in $F$. Let $U_{1}^{\mathrm{ev}}=\left\{z \in U_{1}: l_{1}(z)\right.$ is even $\}$ denote the visible elements of even length. If $\mathrm{ab}^{-1}\left(U_{1}^{\mathrm{ev}}\right)=\left\{v \in V_{1}:|v|_{A}\right.$ is even $\}$, then

$$
\begin{aligned}
\lim _{m \rightarrow \infty} \frac{\gamma_{A}\left(2 m, V_{1}\right)}{\gamma_{A}(2 m, F)} & =2 \rho_{\infty}\left(U_{1}^{\mathrm{ev}}\right)=\frac{2^{r}-2}{\left(2^{r}-1\right) \zeta(r)}, \\
\lim _{m \rightarrow \infty} \frac{\gamma_{A}\left(2 m-1, V_{1}\right)}{\gamma_{A}(2 m-1, F)} & =2 \rho_{\infty}\left(U_{1}\right)-2 \rho_{\infty}\left(U_{1}^{\mathrm{ev}}\right)=\frac{2^{r}}{\left(2^{r}-1\right) \zeta(r)} .
\end{aligned}
$$

Proof. For $c>0$ let $\Omega_{c}:=\left\{x \in \mathbb{R}^{r}:\|x\|<c\right\}$ and let $\overline{\Omega_{c}}$ be the complement of $\Omega_{c}$. Then

$$
\lim _{c \rightarrow \infty} \mathfrak{N}\left(\Omega_{c}\right)=1 .
$$

Let $\varepsilon>0$ be arbitrary. By (4.4) and Proposition 4.8 (b) we can choose $c>0$ such that

$$
\left|\mathfrak{n}\left(\Omega_{c}\right)-1\right| \leq \varepsilon / 3 \quad \text { and } \quad \lim _{n \rightarrow \infty} \sum_{x \in \overline{\Omega_{c}}} p_{n}(x) \leq \varepsilon / 6 .
$$

Let $S$ be a $G_{r}$-invariant subset of $\mathbb{Z}^{r}$. By Theorem 4.9 and the above formula there is some $n_{0} \geq 1$ such that for all $n \geq n_{0}$ we have

and

$$
\left|\sum_{x \in S \cap \sqrt{n} \Omega_{c}} p_{n}(x / \sqrt{n})-\rho_{\infty}(S) \Re\left(\Omega_{c}\right)\right| \leq \varepsilon / 3
$$

$$
\sum_{x \in \overline{\Omega_{c}}} p_{n}(x) \leq \varepsilon / 3
$$

Let

$$
Q(n):=\frac{\gamma_{A}\left(n-1, \mathrm{ab}^{-1}(S)\right)}{2 \gamma_{A}(n-1, F)}+\frac{\gamma_{A}\left(n, \mathrm{ab}^{-1}(S)\right)}{2 \gamma_{A}(n, F)} .
$$

For $n \geq n_{0}$ we let

$$
\begin{aligned}
Q(n)= & \left(\frac{\sharp\left\{g \in F: \mathrm{ab}(g) \in S,|g|_{A}=n-1 \text { and }\|\mathrm{ab}(g)\|<c \sqrt{n}\right\}}{2 \gamma_{A}(n-1, F)}\right. \\
& \left.+\frac{\sharp\left\{g \in F: \mathrm{ab}(g) \in S,|g|_{A}=n \text { and }\|\mathrm{ab}(g)\|<c \sqrt{n}\right\}}{2 \gamma_{A}(n-1, F)}\right) \\
& +\left(\frac{\sharp\left\{g \in F: \mathrm{ab}(g) \in S,|g|_{A}=n-1 \text { and }\|\mathrm{ab}(g)\| \geq c \sqrt{n}\right\}}{2 \gamma_{A}(n-1, F)}\right. \\
& \left.+\frac{\sharp\left\{g \in F: \mathrm{ab}(g) \in S,|g|_{A}=n \text { and }\|\mathrm{ab}(g)\| \geq c \sqrt{n}\right\}}{2 \gamma_{A}(n-1, F)}\right)
\end{aligned}
$$




$$
=\sum_{x \in S \cap \sqrt{n} \Omega_{c}} p_{n}(x / \sqrt{n})+\sum_{x \in S \cap\left(\mathbb{R}^{r} \backslash \sqrt{n} \Omega_{c}\right)} p_{n}(x / \sqrt{n}) .
$$

In the last line of the above equation, by (4.5), the first sum differs from $\rho_{\infty}(S) \Re\left(\Omega_{c}\right)$ by at most $\varepsilon / 3$ since $n \geq n_{0}$, and by (4.6) the second sum is $\leq \varepsilon / 3$ given the choice of $c$ and $n_{0}$. Therefore, again by the choice of $c$, we have $\left|Q(n)-\rho_{\infty}(S)\right| \leq \varepsilon$. Since $\varepsilon$ is arbitrary, this proves (i).

We now prove (ii). First notice that since $U_{1}$ is $\operatorname{SL}(r, \mathbb{Z})$-invariant, it is also $G_{r}$ invariant. We check that $U_{1}^{\mathrm{ev}}$ is $G_{r}$-invariant as well. Let $u \in \mathbb{Z}$. Then $u \in U_{1}^{\mathrm{ev}}$ if and only if $\sum_{1 \leq i \leq r}(u)_{i} \bmod 2=0$ and $\operatorname{gcd}(u)=1$. Let $M \in G_{r}$. As $M \in \operatorname{SL}(r, \mathbb{Z})$, $\operatorname{gcd}(M u)=\operatorname{gcd}(u)=1$. Also, as $M=I_{r}$ in $\operatorname{SL}(r, \mathbb{Z} / 2 \mathbb{Z})$,

$$
\sum_{1 \leq i \leq r}(M u)_{i} \bmod 2=\sum_{1 \leq i \leq r}(u)_{i} \bmod 2=0 .
$$

Hence, $U_{1}^{\text {ev }}$ is $G_{r}$-invariant.

We now take $S=U_{1}^{\text {ev }}$, for $n \geq 2$ even. Then

$$
Q(n)=\frac{\gamma_{A}\left(n-1, \mathrm{ab}^{-1}\left(U_{1}^{\mathrm{ev}}\right)\right)}{2 \gamma_{A}(n-1, F)}+\frac{\gamma_{A}\left(n, \mathrm{ab}^{-1}\left(U_{1}^{\mathrm{ev}}\right)\right)}{2 \gamma_{A}(n, F)}=\frac{\gamma_{A}\left(n, \mathrm{ab}^{-1}\left(U_{1}^{\mathrm{ev}}\right)\right)}{2 \gamma_{A}(n, F)} .
$$

The latter equality follows from the fact that $\mathrm{ab}^{-1}\left(U_{1}^{\mathrm{ev}}\right)=\left\{v \in V_{1}:|v|_{A}\right.$ is even $\}$.

By (i),

$$
\lim _{m \rightarrow \infty} \frac{\gamma_{A}\left(2 m, V_{1}\right)}{\gamma_{A}(2 m, F)}=2 \lim _{m \rightarrow \infty} Q(2 m)=2 \rho_{\infty}\left(U_{1}^{\mathrm{ev}}\right) .
$$

Thus $\lim _{m \rightarrow \infty} \frac{\gamma_{A}\left(2 m-1, V_{1}\right)}{\gamma_{A}(2 m-1, F)}=2 \rho_{\infty}\left(U_{1}\right)-2 \rho_{\infty}\left(U_{1}^{\text {ev }}\right)$. By Proposition 3.1 and Proposition 3.5, we obtain the desired results.

We now focus on surface and free groups.

Corollary 4.11. Let $k \geq 2$ and let $F$ be a free group of rank $k$ or a surface group of genus $k$. Let $A$ and $r$ be as in Notation 4.1. Then

(i) $\lim _{m \rightarrow \infty} \frac{\gamma_{A}\left(2 m, V_{1}\right)}{\gamma_{A}(2 m, F)}=\frac{2^{r}-2}{\left(2^{r}-1\right) \zeta(r)}$,

(ii) $\lim _{m \rightarrow \infty} \frac{\gamma_{A}\left(2 m-1, V_{1}\right)}{\gamma_{A}(2 m-1, F)}=\frac{2^{r}}{\left(2^{r}-1\right) \zeta(r)}$.

Proof. By Theorem 4.2, $F, A$ and $r$ satisfy the hypotheses of Theorem 4.10. It only remains to show that $\mathrm{ab}^{-1}\left(U_{1}^{\mathrm{ev}}\right)=\left\{v \in V_{1}:|v|_{A}\right.$ is even $\}$. Let $f$ be an element of $F$ such that $\operatorname{ab}(f)=0 \in \mathbb{Z}^{r}$. Then any word representing $w$ has the same number of $a$ and $a^{-1}$ and thus it has even length.

Since ab maps elements of $A$ to unit vectors, for $u \in U_{1}^{\text {ev }}$ there exists $v \in$ $\mathrm{ab}^{-1}\left(U_{1}^{\text {ev }}\right)$ of even length. If $\mathrm{ab}(v)=\mathrm{ab}\left(v^{\prime}\right)$, then $\mathrm{ab}\left(v^{\prime} v^{-1}\right)=0$. Hence $v^{\prime} v^{-1}$ has even length, and so does $v^{\prime}$. Thus Theorem 4.10 (ii) applies. 
On the asymptotics of visible elements and homogeneous equations in surface groups 635

\section{Asymptotic behavior of homogeneous equations in surface groups}

We now study the asymptotic behavior of $e_{\gamma}\left(G_{n}, G_{k}, s, t\right)$ when $G_{n}$ and $G_{k}$ are surface or free groups, or more generally, satisfy the hypothesis of Theorem 4.10(ii).

Theorem 5.1. Let $G_{k}$ and $G_{n}$ be free or surface groups and let $A, B$ be their respective generating sets, as in Notation 4.1. Let $r(k)$ and $r(n)$ denote the ranks of the abelianization of $G_{k}$ and $G_{n}$, respectively. Let $\varepsilon, \delta \in\{0,1\}$. Then the following inequalities hold:

$$
\begin{gathered}
\frac{2^{r(n)}-2(1-\varepsilon)}{\left(2^{r(n)}-1\right) \zeta(r(n))} \leq \liminf _{s \rightarrow \infty, t \rightarrow \infty} e_{\gamma}\left(G_{n}, G_{k}, 2 s+\varepsilon, 2 t+\delta\right), \\
\limsup _{s \rightarrow \infty, t \rightarrow \infty} e_{\gamma}\left(G_{n}, G_{k}, 2 s+\varepsilon, 2 t+\delta\right) \\
\quad \leq 1-\frac{2^{r(k)}-2(1-\delta)}{\left(2^{r(k)}-1\right) \zeta(r(k))}\left(1-\frac{2^{r(n)}-2(1-\varepsilon)}{\left(2^{r(n)}-1\right) \zeta(r(n))}\right) .
\end{gathered}
$$

Proof. Let $V_{t}$ and $W_{t}$ denote the sets of $t$-visible elements in $G_{n}$ and $G_{k}$, respectively. Let

$$
\begin{array}{r}
E(s, t)=\left\{(u, v) \in G_{n} \times G_{k}:|u|_{A}=s,|v|_{B}=t, \phi(u)=v\right. \\
\text { for some } \left.\phi \in \operatorname{Hom}\left(G_{n}, G_{k}\right)\right\} .
\end{array}
$$

Then $e_{\gamma}\left(G_{n}, G_{k}, s, t\right)=\frac{|E(s, t)|}{\gamma_{B}\left(s, G_{n}\right) \gamma_{A}\left(t, G_{k}\right)}$.

By Lemma 3.3 we have the following inequalities:

$$
\gamma_{B}\left(s, W_{1}\right) \gamma_{A}\left(t, G_{k}\right) \leq|E(s, t)| \leq \gamma_{B}\left(s, G_{n}\right) \gamma_{A}\left(t, G_{k}\right)-\sum_{r \neq 1} \gamma_{B}\left(s, W_{r}\right) \gamma_{A}\left(t, V_{1}\right) \text {. }
$$

The left inequality holds because every element $v$ in $G_{k}$ is the homomorphic image of a visible element in $G_{n}$. The right inequality holds because no visible element in $G_{k}$ is the homomorphic image of an $r$-visible element in $G_{n}$ if $r \neq 1$.

By dividing both sides by $\gamma_{B}\left(s, G_{n}\right) \gamma_{A}\left(t, G_{k}\right)$, we get

$$
\frac{\gamma_{B}\left(s, W_{1}\right)}{\gamma_{B}\left(s, G_{n}\right)} \leq e_{\gamma}\left(G_{n}, G_{k}, s, t\right) \leq 1-\frac{\sum_{r \neq 1} \gamma_{B}\left(s, W_{r}\right) \gamma_{A}\left(t, V_{1}\right)}{\gamma_{B}\left(s, G_{n}\right) \gamma_{A}\left(t, G_{k}\right)}=f(s, t),
$$

where

$$
f(s, t)=1-\frac{\gamma_{A}\left(t, V_{1}\right)}{\gamma_{A}\left(t, G_{k}\right)} \frac{\gamma_{B}\left(s, G_{n}\right)-\gamma_{B}\left(s, W_{1}\right)}{\gamma_{B}\left(s, G_{n}\right)} .
$$

Let us use $\beta_{m, k}$ to denote the limits, which depend on the parity of $m$ and the rank of the abelianization of $G_{n}$ and $G_{k}$, found in Corollary 4.11. That is, $\beta_{m, k}=\frac{2^{r(k)}-2}{\left(2^{r(k)}-1\right) \zeta(r(k))}$ if $m$ is even, and $\beta_{m, k}=\frac{2^{r(k)}}{\left(2^{r(k)}-1\right) \zeta(r(k))}$ if $m$ is odd. In order to simplify the exposition 
we will abuse the fact that $\beta_{m, k}$ depends on the parity of $m$ and for the next paragraph ignore the parities of $s$ and $t$.

Then

$$
\lim _{s \rightarrow \infty, t \rightarrow \infty} f(s, t)=1-\beta_{t, k}\left(1-\beta_{s, n}\right),
$$

and we get the inequalities

$\beta_{s, n} \leq \liminf _{s \rightarrow \infty, t \rightarrow \infty} e_{\gamma}\left(G_{n}, G_{k}, s, t\right) \leq \limsup _{s \rightarrow \infty, t \rightarrow \infty} e_{\gamma}\left(G_{n}, G_{k}, s, t\right) \leq 1-\beta_{t, k}\left(1-\beta_{s, n}\right)$.

Now taking into account the parities of $s$ and $t$ we get the inequalities in the statement of the theorem.

Thus the probability of an $(s, t)$-homogeneous equation to be satisfiable is neither 0 nor 1 as $s, t$ go to infinity. One sees this by choosing $G_{n}$ to be the free group on $n$ generators and $G_{k}$ a surface group of genus $g \geq 2$ or a free group of rank $\geq 2$ in Theorem 5.1.

Corollary 5.2. Let $G$ be a surface group of genus $g \geq 2$ or a free group of rank $\geq 2$. Let

$$
A(s, t)=\frac{\sharp\{\text { satisfiable }(s, t) \text {-homogeneous equations in } G \text { in } n \text { variables }\}}{\sharp\{(s, t) \text {-homogeneous equations in } G \text { in } n \text { variables }\}} .
$$

Then

$$
0<\liminf _{s \rightarrow \infty, t \rightarrow \infty} A(s, t) \leq \limsup _{s \rightarrow \infty, t \rightarrow \infty} A(s, t)<1 .
$$

Similarly, by choosing both $G_{n}$ and $G_{k}$ in Theorem 5.1 to be surface groups one obtains the following.

Corollary 5.3. Let $\Sigma$ be an orientable closed surface of genus $k \geq 2$. We fix a presentation for $\pi_{1}(\Sigma),\left\langle a_{1}, b_{1}, \ldots, a_{k}, b_{k} \mid\left[a_{1}, b_{1}\right] \ldots\left[a_{k}, b_{k}\right]\right\rangle$. For a closed curve $\gamma$ in $\Sigma$ we denote by $[\gamma]$ the image of $\gamma$ in $\pi_{1}(S)$ and by $|[\gamma]|$ the length of $[\gamma]$ with respect to $\left\{a_{1}, b_{1}, \ldots, a_{k}, b_{k}\right\}$.

We say that $\gamma_{2}$ is the image of $\gamma_{1}$ if it is the image of $\gamma_{1}$ under a continuous map $S \rightarrow S$.

Let

$$
B(s, t)=\frac{\sharp\left\{\left(\left[\gamma_{1}\right],\left[\gamma_{2}\right]\right) \in \pi_{1}(S)^{2},\left(\left|\left[\gamma_{1}\right]\right|,\left|\left[\gamma_{2}\right]\right|\right)=(s, t) \text { with } \gamma_{2} \text { the image of } \gamma_{1}\right\}}{\sharp\left\{\left(\left[\gamma_{1}\right],\left[\gamma_{2}\right]\right) \in \pi_{1}(S)^{2},\left(\left|\left[\gamma_{1}\right]\right|,\left|\left[\gamma_{2}\right]\right|\right)=(s, t)\right\}} .
$$

Then

$$
0<\liminf _{s \rightarrow \infty, t \rightarrow \infty} B(s, t) \leq \limsup _{s \rightarrow \infty, t \rightarrow \infty} B(s, t)<1 .
$$


On the asymptotics of visible elements and homogeneous equations in surface groups 637

Thus for a fixed orientable surface $\Sigma$, the probability of a closed curve in $\Sigma$ to be the image of another closed curve in $\Sigma$ by a continuous map is neither 0 nor 1 as the curves get more and more "complicated."

Acknowledgments. We are grateful to Fernando Chamizo for helpful conversations. The first-named author was jointly funded by the MEC (Spain) and the EFRD (EU) through Projects MTM2006-13544 and MTM2008-01550. The second-named author was partially supported by the SNF (Switzerland) through project number 200020113199 and by the Marie Curie Reintegration Grant 230889. The third-named author was supported by the grant MEC-FEDER Ref. MTM2009-08869 from the Dirección General de Investigación, MEC (Spain).

\section{References}

[1] G. N. Arzhantseva and A. Yu. Ol'shanskii, The class of groups all of whose subgroups with lesser number of generators are free is generic. Mat. Zametki 59 (1996), 489-496; English transl. Math. Notes 59 (1996), 350-355. Zbl 0877.20021 MR 1445193

[2] P. Billingsley, Probability and measure. 2nd ed., John Wiley, New York 1986. Zbl 0649.60001 MR 830424

[3] D. Calegari and K. Fujiwara, Combable functions, quasimorphisms, and the central limit theorem. Ergodic Theory Dynam. Systems 30 (2010), 1343-1369. Zbl 1217.37025 MR 2718897

[4] J. Christopher, The asymptotic density of some $k$-dimensional sets. Amer. Math. Monthly 63 (1956), 399-401. Zbl 0070.04101 MR 0097363

[5] L. Ciobanu, Polynomial-time complexity for instances of the endomorphism problem in free groups. Internat. J. Algebra Comput. 17 (2007), 289-328. Zbl 1125.20018 MR 2310149

[6] N. M. Dunfield and D. P. Thurston, A random tunnel number one 3-manifold does not fiber over the circle. Geom. Topol. 10 (2006), 2431-2499. Zbl 1139.57018 MR 2284062

[7] R. H. Gilman, A. Myasnikov, and V. Roman'kov, Random equations in free groups. Groups Complex. Cryptol. 3 (2011), 257-284. Zbl 06036193 MR 2898893

[8] R. H. Gilman, A. Myasnikov, and V. Roman'kov, Random equations in nilpotent groups. J. Algebra 352 (2012), 192-214. Zbl 06055217 MR 2862182

[9] I. Kapovich, I. Rivin, P. Schupp, and V. Shpilrain, Densities in free groups and $\mathbb{Z}^{k}$, visible points and test elements. Math. Res. Lett. 14 (2007), 263-284. Zbl 1132.20017 MR 2318624

[10] I. Kapovich, P. Schupp, and V. Shpilrain, Generic properties of Whitehead's algorithm and isomorphism rigidity of random one-relator groups. Pacific J. Math. 223 (2006), 113-140. Zbl 1149.20028 MR 2221020

[11] Y. N. Petridis and M. S. Risager, Equidistribution of geodesics on homology classes and analogues for free groups. Forum Math. 20 (2008), 783-815. Zbl 1166.53015 MR 2445118 
[12] I. Rivin, Growth in free groups (and other stories) - twelve years later. Illinois J. Math. 54 (2010), 327-370. Zbl 1225.05128 MR 2776999

[13] R. Sharp, Local limit theorems for free groups. Math. Ann. 321 (2001), 889-904. Zbl 1012.20019 MR 1872533

[14] W. Woess, Cogrowth of groups and simple random walks. Arch. Math. (Basel) 41 (1983), 363-370. Zbl 0522.20043 MR 731608

[15] R. J. Zimmer, Ergodic theory and semisimple groups. Monogr. Math. 81, Birkhäuser, Basel 1984. Zbl 0571.58015 MR 776417

Received September 6, 2010; revised May 9, 2011

Y. Antolín, University of Southampton, Building 54, Highfield, Southampton, SO17 1BJ, United Kingdom

E-mail: Y.Antolin-Pichel@ soton.ac.uk

L. Ciobanu, Institut de Mathématiques, Université de Neuchâtel, Rue Emile-Argand 11, 2000 Neuchâtel, Switzerland

E-mail: laura.ciobanu@unine.ch

N. Viles, Departament de Matemàtiques, Universitat Autònoma de Barcelona, 08193 Bellaterra (Barcelona), Spain

E-mail: nviles@mat.uab.cat 